\title{
3. Building knowledge futures for cerebral palsy: examples from The Spastic Centre
}

\author{
Robyn Cummins
}

\section{Introduction}

The knowledge-brokering field has traditionally involved a triad of stakeholders: researchers, practitioners and policymakers. The Spastic Centre has a record of successful knowledge sharing and brokering in these realms, but is increasingly aware of two additional stakeholder groups with a growing influence in the field: consumers and the corporate sector. The Spastic Centre is an organisation attuned to the benefits of knowledge brokering and is now engaged in activities involving all five of these groups.

\section{Background}

Cerebral palsy is a permanent physical condition that affects movement. There are approximately 11000 people in New South Wales with cerebral palsy and an estimated 33797 across Australia (in 2007). The impact of cerebral palsy is significant, with considerable social and economic costs to the family and the individual (Access Economics 2008).

People with cerebral palsy can have seizures and other impairments affecting their speech, vision, hearing and/or intellect. Worldwide, the incidence of cerebral palsy is the same - one in every 400 births - and, for most, the cause is unknown.

The Spastic Centre offers services to children and adults with cerebral palsy and allied conditions. These services include physiotherapy, speech pathology, occupational therapy, technology, respite, accommodation support, family support, recreation, employment, equipment, recreation, day services and mobility programs. 
Bridging the 'Know-Do' Gap

The organisation also provides education, consultancy and web-based information to clients, the community, service providers and the research and education sectors across Australia and internationally.

The centre has approximately 850 staff and 1000 volunteers at 70 service sites across New South Wales and the Australian Capital Territory.

\section{Knowledge and cerebral palsy}

People with cerebral palsy, their families, carers and service providers are on a lifelong quest for knowledge. On diagnosis, families first ask, 'Why did this happen?', then, 'What does the future hold for my child?', then, 'How can I make the best life for my child?' At every stage of their child's life, the family's knowledge needs change. Critical times are around transition points: adjusting to the diagnosis, entering the service system, preschool and school and the transition to adulthood.

No less important are the needs of the growing child - from the time they first ask questions about their disability through to making decisions about their lives as emerging adults. Throughout their adult life, people with cerebral palsy need knowledge of the services and supports that will enable them to lead the best possible life.

Service providers need sound evidence about the interventions that are best suited to each individual. Providers often act as intermediaries, supporting their clients and families by filtering and interpreting the vast array of conflicting information that is increasingly available.

In all instances, a combination of explicit knowledge and tacit knowledge is required

- explicit knowledge: codified knowledge that can be transmitted in formal, systematic language and shared in the form of documents, data, manuals, and so on

- tacit knowledge: personal, context-specific knowledge that is difficult to formalise, record or articulate.It is deeply rooted in individuals' actions and experience as well as in the ideals, values or emotions they embrace (Kidwell et al. 2000). 


\section{Knowledge brokering}

Knowledge sharing is a multi-layered process of exchanging knowledge among individuals and between sectors. Knowledge brokering encompasses a range of formal and informal activities and processes undertaken to establish relationships and facilitate effective knowledge exchange (Canadian Health Services Research Foundation n.d.).

The activities of a knowledge broker can include

- developing an understanding of the needs of the different sectors and identifying opportunities for linkages

- bringing individuals and sectors together in knowledge networks to exchange information and work together

- helping groups communicate and understand each other's needs and abilities

- mapping, capturing, translating and distilling knowledge for exchange

- providing the tools for others to capture, transfer, exchange and collaborate around knowledge

- identifying and disseminating best practice

- promoting the use of evidence in funding, planning and delivering services.

Knowledge brokers are entrepreneurial; they are networkers, problem solvers and innovators. They are clear communicators and are perceived as trusted and credible individuals. The role requires an understanding of the cultures of the different environments and they must be able to find and assess relevant knowledge, including research evidence. A knowledge broker is a facilitator, a mediator and a negotiator. They will also love learning and sharing new knowledge (Clarke and Kelly 2005; Canadian Health Services Research Foundation 2003).

Above all, knowledge brokers recognise that knowledge transfer and exchange is a social activity. 'Knowledge depends for its circulation on interpersonal networks, and will only diffuse if these social features are taken into account and barriers overcome' (Greenhalgh et al. 2004:607). 


\section{Traditional forms of knowledge brokering at The Spastic Centre}

For The Spastic Centre, the traditional sectors or stakeholder groups in the knowledge-sharing and brokering process have comprised: researchers, practitioners (service providers - internal and external) and government policymakers/funders.

The Spastic Centre takes a leadership role within the sector, with a range of knowledge-sharing and brokering initiatives among the research, policy and practitioner communities.

The organisational attributes that contribute to this include

- a culture of innovation, to develop new and creative solutions

- an acknowledgment of the value of every individual and of groups

- a family-centred framework of service delivery, with a strengths-based approach

- an ethos of evidence-based service delivery

- an outward-looking perspective - drawing ideas from a spectrum of sectors

- a record of cultivating national and international networks for support and knowledge sharing

- a strong desire to form partnerships for the benefit of clients and the sector

- a commitment to positively influence the actions, policies and attitudes of government, business and the community.

In recent years, two major projects conducted by The Spastic Centre have strongly utilised knowledge brokering - the first to achieve significant changes in organisational practice and the second in government policy. Both engaged with traditional research/practice/policy stakeholders.

The first project - conducted by The Cerebral Palsy Institute, the research arm of The Spastic Centre - used knowledge brokering to transfer research evidence into practice. This resulted in significant changes to organisational culture, employees' knowledge and management's framework of thinking.

The second project followed the path of service innovation to research, the results of which were brokered to government to achieve changes in public policy. 


\section{Research to practice}

\section{Evidence-based practice: education with employer support}

The following section was written by Dr Iona Novak and describes an example of a knowledge-brokering technique, which was part of a study conducted at The Spastic Centre by staff from The Cerebral Palsy Institute. The study is published elsewhere (Novak et al. 2008; Novak and McIntyre forthcoming).

The 2001-2004 Strategic Plan of The Spastic Centre included a strategy to, 'Introduce an evidence-based approach across all services'. The first stage was to create a culture of research and evidence-based practice within the organisation.

At the commencement of the project, three stakeholders were identified: clients; staff as providers of service; and managers, as decision-makers and leaders, and as the interface between the organisation, government and donors. It was recognised that each group had different needs and strengths, requiring different knowledge strategies.

The knowledge brokering and transfer activities that assisted the cultural shift included:

- Discussions with allied health staff and management about evidencebased practice and its application within a community-based disability service;

- Working with management and the Health Services Union to embed evidence-based practice into allied health role descriptions and linking advanced demonstration of behaviours to remuneration and incentives for promotion; and

- Preparing and presenting a business case to resource internal clinical consultants to take a leadership role in the rollout of evidence-based practice within the organisation, support the knowledge sharing activities and act as evidence-based practice knowledge brokers.

From 2004, the emphasis shifted from changing organisational culture to underpinning all services with evidence. The dual interventions selected to progress this were: knowledge and skill development; and employer support strategies. A range of knowledge sharing and brokering activities were undertaken to support this intervention. 
Bridging the 'Know-Do' Gap

Knowledge and skill development - two key areas were targeted: evidence-based practice and outcome measurement. The two topics are closely interlinked and as a consequence, two parallel research projects were initiated as part of the approach.

Activities included:

- A two-day competency-based training for senior clinical staff on leadership, mentoring and evidence-based practice skills;

- Professional development for junior clinicians led by senior staff and ongoing professional development opportunities under the banner of the CP Institute;

- Development of discipline-specific networks for peer support;

- Support for staff to develop a set of critically appraised topics (CATs) on common interventions; and

- Provision of an evidence alert system to assist practitioners when selecting an intervention to meet the goals of a client or family.

Employer support was also offered. Consultations were held with management to ensure that the activities necessary to implement evidence-based practice and outcome measurement across the organisation were supported and encouraged. Management was also supported to write an interface between evidence-based practice and outcome measurement to embed it, not just as a professional activity, but into service offerings, systems and the processes of service delivery. Senior allied health (Level 3) clinicians were also appointed to take a mentoring role to foster evidence-based practice behaviours across the organisation.

A comprehensive literature review was undertaken at the beginning of the project and two international experts-Margaret Wallen of the Children's Hospital Westmead and Annie McCluskey of the University of Sydney-were consulted about local (NSW) factors that could influence the uptake of evidencebased practice.

Three research projects also ran in parallel with the initiative. Julia Bowman from the University of Western Sydney conducted a randomised control trial that investigated whether education and follow-up mentoring influenced whether or not health professionals used outcomes measurement in practice. Staff members were offered a one-day workshop and follow-up mentoring designed to help them adopt outcome measurement as part of routine practice. Anne Cusick from the University of Western Sydney led two research projects. The first investigated what were the optimal professional development standards recommended in the 
literature and how the activities of The Spastic Centre compared. The second question examined which occupational-therapy interventions for people with cerebral palsy had the highest levels of research evidence in the literature and how this matched the interventions offered by the organisation. Results from these studies are available in the published literature (Cusick et al. 2009).

Encouragingly, staff at all levels are now taking on roles as catalysts and knowledge brokers - supporting others by sharing knowledge and engaging in collaborative problem solving in mentoring sessions and in internal clinical email groups.

Members of The Spastic Centre's Cerebral Palsy Institute now also provide extensive brokering in the form of consultations and education on the topics of behaviour change in evidence-based practice and outcome measurement to health practitioners and managers in other organisations.

\section{Practice to research to policy}

\section{Intensive family support options}

The Spastic Centre has a strong record of service innovation. Often, a research project will accompany a new service development to provide sound evidence on its outcomes and effectiveness. With these findings, the knowledge can be shared with the disability service sector and approaches can be made to policymakers and potential funders to extend the offerings of these services across the sector.

One successful example was the Intensive Family Support Options (IFSO) service. The Spastic Centre first offered this service for families who were under such stress that they were requesting an out-of-home placement for their child with a disability. The NSW Government supported the first program under innovative respite service funding.

The service aimed to keep families safely together - with consideration for their physical and emotional wellbeing. The program elements unique to this service were

- short-term (three-month) intensive support

- one therapist worked with each family and was available 24 hours a day and seven days a week, if necessary

- a solution-focused, strengths-based, family-centred (rather than childcentred) approach 
Bridging the 'Know-Do' Gap

- the therapist had a small caseload and offered very practical support

- brokerage funding was available for small household purchases that would make a difference in the families' day-to-day lives (Baldry et al. 2005).

In partnership with the University of New South Wales' School of Social Work, IFSO received an Australian Research Council (ARC) grant to determine 'whether the IFSO program significantly and positively influenced outcomes for the child and family, and if significant positive change did occur, which program elements, strategies and/or techniques significantly contributed to positive client outcomes'.

The study affirmed that the interventions improved families' levels of wellbeing and functioning and were significantly successful in reducing child abuse potential (Baldry et al. 2005).

To extend the knowledge transfer, the IFSO team and the research team from the University of New South Wales presented the findings and service model at a number of national and international conferences and to all referring agencies. They also had extensive consultations with the state government about the effectiveness of the program. In 2005-06, the NSW Government announced funding of \$4.1 million under the Intensive Family Support Initiative to establish new services in every region.

It was fortunate that the timing of the brokering efforts coincided with a growing realisation within the disability sector and the government that it was time for a change. Residential facilities were closing and there was increasing recognition that children were better off with their families. The question then became 'how could children be kept safely in the family home'? Although some in the sector had reservations, the strengths-based, solution-focused philosophy and program elements of IFSO were overwhelmingly welcomed as a positive change in approach.

The IFSO project was an example of successful brokering of knowledge about a program - backed by evidence - to policymakers, and now benefiting families across the state.

\section{New landscape of knowledge brokering}

In the past five years, The Spastic Centre has recognised the influence of two new stakeholders in the knowledge-brokering arena: consumers and corporations. 


\section{The rise of the consumer on the Web}

Australians were early adopters of the Internet. Now, 72 per cent of households have home Internet access. Broadband is accessed by 62 per cent of all households in Australia and 86 per cent of all households with Internet access (ABS 2009).

For many years, the World Wide Web has been a source of health information for consumers. In 2000, the Pew Internet and American Life Project reported the Internet's powerful influence on 'health seekers': 55 per cent said that access to the Internet had improved the way they received medical and health information, while 47 per cent of those who sought health information for themselves during their last online search said the material affected their decisions about treatment and care. By 2006, Pew reported that 80 per cent of adults in the United States with Internet access used it for healthcare purposes.

Since 2000, the evolution in communications enabled by the Internet has changed the landscape of knowledge transfer and brokering to and between consumers. In recent years, the Web has changed from an 'expert' model, where information and knowledge were part of a one-way transfer from expert to consumer. Colloquially known as Web 2.0, the Web is now

- social: it is a platform for connecting people

- participatory: the consumer has become the content creator and this is shared peer to peer

- based on groups: formal and informal groups are now easily formed to share information and create new knowledge; many individuals can make small contributions for the greater good

- democratic: web initiatives often have no formal leadership, but rely on catalysts who empower others and then 'leave it to the crowd' (Brafman and Beckstrom 2006)

- global: there is a blurring of national, cultural and sectoral boundaries on the Web (Friedman 2005).

The Web is now the world's largest social network. The Internet has enabled social interactions that go beyond the traditional venues of one's community, work and home settings (Snyder et al. 2006). In 2008, audience measurement company ComScore estimated that the Web had a worldwide audience of 190 858000 people, while the audience for social networking sites was 131808000 . In 2007, Technorati was also tracking 70 million weblogs, with approximately 120000 new weblogs created daily (Sifry 2007). 
Yet, as Forkner-Dunn (2003) contends, '[m]ost institutions funding medical research, health policymakers, and health care professionals have ignored both the "e-revolution" and the fact that it is consumer driven'.

A study by Sillence et al. (2006) compared the changes in online health usage between 2000 and 2005. The most popular health topic in 2000 was cancer, while in 2005 it was alternative medicine- ' $[a]$ topic little discussed in mainstream healthcare and one which has found a niche online' (Sillence et al. 2006:404). The results also show that people are increasingly seeking personalised health advice. 'Health consumers are searching for sites that match their own social "identity", sites which they feel they can relate to and that are "written for people like themselves"' (Sillence et al. 2006:398).

\section{Changes to The Spastic Centre's approach}

Observations of this web-enabled consumer movement influenced The Spastic Centre's 2007-10 strategic plan. The plan includes two outcomes: an expanded range of options for clients and families to connect and reduce isolation, and families which are informed about the best service choice offerings.

Before the development of the plan, a number of online social networking platforms had been identified-blogs, discussion forums, special-interest groups and wikis - where parents of children with a disability not only were connecting with one another for mutual support but appeared to be strongly influencing each other about intervention choices, many of which were not based on evidence. Those engaging in alternative therapies are often very vocal about the perceived benefits for their child. Their credibility is based on the fact that they are not professionals, whose practice is grounded in evidence, but rather parents experiencing the same journey as their readers.

In this way, consumers are increasingly making decisions about interventions outside of the evidence-based research/practice/policy realms. Knowledge about this phenomenon was brokered to senior management in the formative stages of the strategic plan. The brokering process involved the identification of web trends, filtering and synthesising this information in light of the expressed needs of the organisation's client base, presentations to senior management and individual negotiation with key managers before the strategic planning process.

The organisation began to consider ways in which parents could be offered mutual support and connection, allowing the 'authentic voice' to be heard while ensuring that the evidence of the best interventions was communicated. Unlike one-way knowledge transfer, this involves knowledge brokering: building personal relationships, facilitating dialogue and debate, understanding the issues for all parties and translating the message for the different audiences. 


\section{CP Blogs}

A recent major initiative to address this issue is CP Blogs $(<$ www.cpblogs.org. $\mathrm{au}>$ ). The Spastic Centre has developed it as a place for people with cerebral palsy, their family and friends to come together to share their experiences.

There are four blogs

- Hey Dad: written by a father of a child with cerebral palsy, about the day-today challenges and unexpected bonuses of having a child with a disability, written from a strengths-based perspective

- Freefall: about moving out of home and making a life in the city as a young woman with cerebral palsy; this age group was targeted as many young people have difficulty gaining independence in the transition to adult life

- Web2Go: written by a young journalist with cerebral palsy about the technologies and opportunities for engagement on the Web for people with cerebral palsy; in many ways, the Web affords great social potential for people with a disability, as the barriers to physical access and obstacles for people who are non-verbal are less evident in this sphere

- The Scene: features news, activities, resources and new research for people with cerebral palsy and their families; The Scene was formerly an e-newsletter with a large number of Australian readers.

The blogs are all designed to build a community of readers and to provide quality information for the benefit of people with cerebral palsy and their families. Most importantly, they facilitate the brokering of tacit and explicit knowledge through the blog posts and the provision of a comments function. Knowledge brokering is about conversations. Readers are able to identify with the bloggers as they understand the dichotomy of 'heart' and 'head' that is the journey of cerebral palsy. Readers and bloggers can engage with one another to share their thoughts and debate different perspectives.

The bloggers were all selected for their 'authenticity' (that is, they are people with cerebral palsy or a parent), their ability to write entertainingly and with great insight and their positive, strengths-based approach.

Production standards for the blogs, including design and content, are of a high standard - a barometer of a site's perceived trustworthiness (Sillence et al. 2005). By building a personal reputation in the 'blogosphere', the bloggers might be able to positively influence discussion in online spaces that have not traditionally been the realm of service providers. With trust comes credibility 
and the influence that this can bring. The Web is filled with proponents of interventions that are not based on evidence. The blog initiatives could assist to redress the balance.

The blogs are also a way to support practitioners to truly understand the issues faced by people with cerebral palsy and their families in a safe (that is, arm'slength) environment. The bloggers are already expressing their opinions about what they find helpful in practice and practitioners have begun to engage in the dialogue through the comments field.

The blogs are linked from The Spastic Centre's 'About cerebral palsy' page, which has an exceptionally high Google results rating: number one in an Australian search for the phrase 'cerebral palsy' and number two in a worldwide search for the phrase. This will attract traffic to the blog site and assist in the communication of the evidence-based messages.

By facilitating knowledge sharing and brokering among people with cerebral palsy and their families, and with practitioners, The Spastic Centre can make one of its most valuable contributions to the lives of its clients. Brokering knowledge gleaned from the 'authentic voice' to practitioners, researchers, policymakers and funders will be a way of placing on the agenda some of the issues that most impact the day-to-day lives of people with cerebral palsy and their families.

\section{Corporate social responsibility}

In Australia, corporate community investment is increasingly regarded as a core business activity. In a study reported in Corporate Community Investment in Australia (2007), the reasons for this include

- to win and maintain community trust

- to be seen as an employer of choice by staff, particularly young staff who are a major and growing driver of corporate community investment activity

- to broaden the understanding and perspectives of managers and staff

- to build relationships with key stakeholders, including corporate critics.

Sharing and brokering knowledge about the needs and experiences of clients and their families are important ways for not-for-profit organisations to harness support and build partnerships with the corporate sector. 


\section{The Spastic Centre's involvement with the corporate sector}

The Spastic Centre has strong support from the Australian corporate sector in the areas of service innovation, fundraising for research and voluntary assistance. A number of service developments have been piloted with funding from the sector.

The personal relationships that are developed by the organisation through these networks have led to a number of opportunities to broker knowledge about the needs of people with cerebral palsy that have led to successful outcomes for all stakeholders. High-quality data are matched by personal stories, which can have a powerful impact and are fundamental tools for knowledge brokers (Denning 2006).

\section{Equipment needs and corporate funding}

Equipment - such as wheelchairs, walkers and communication devices - is crucial in reducing the impact of cerebral palsy, allowing children and adults to communicate with friends and family, attend school, participate in their community and find employment. In Australia, the cost of meeting therapy and equipment needs has been estimated to be as high as $\$ 54.8$ million (Australian Institute of Health and Welfare 2006). Although state governments are the largest funders of equipment, there is significant unmet need, with families often seeking funding through community service clubs, small charities or personal fundraising. In 2004, The Spastic Centre began collecting data from its therapists on the unmet need for equipment, including prescription times, waiting lists and outcomes.

At the same time, a major multinational corporate foundation expressed interest in raising money for essential services that would make a significant impact in the lives of people with cerebral palsy.

Knowledge of the unmet need for equipment was brokered to the foundation, together with personal stories of the impact that this was having on the lives of people with cerebral palsy and their families. To address this need, the foundation began an annual fundraising event: a triathlon-style competition between teams comprising some of Australia's major corporations. Since 2004, the event has raised \$2 million, which has funded hundreds of items of equipment for children with cerebral palsy.

The partnership and the data on unmet need have drawn the attention of the NSW Government and in 2008 it augmented the funds raised in the event with a $\$ 220000$ donation. 
Corporate Australia is a powerful ally in addressing the needs of people with a disability. Its increasing interest in practical demonstrations of social responsibility can have direct and systemic benefits. The disability services sector has a role in brokering knowledge about the needs of people with a disability by building continuing relationships with corporations and foundations. Their assistance can take the form of fundraising, volunteering and even knowledge brokering themselves within the corporate and government sectors.

\section{The way ahead}

The Spastic Centre is in a unique position to engage in a range of knowledgebrokering activities for the benefit of people with cerebral palsy and their families. A global perspective and a culture of innovation have allowed the organisation to recognise and respond rapidly to the growing influence of consumer and corporate stakeholders among the traditional pillars of research, practice and policy.

With each group, the centre employs a range of knowledge-brokering activities, including

- development of an understanding of the needs of the different sectors and identifying opportunities for linkages

- helping groups communicate and understand each other's needs

- mapping, capturing, translating and distilling knowledge for exchange

- identifying and disseminating best practice

- promoting the use of evidence in funding, planning and delivering services.

The organisation's ability to respond to the changing needs of its stakeholders has been enhanced by its receptivity to information and communications technology, particularly the opportunities it offers for early observation of trends and for knowledge-brokering activities.

Knowledge brokers must be open to values, concepts and exchanges outside the security of their own field. It is crucial to maintain a watching brief on all sectors of the economy and society in order to engage with the ideas and cultivate the diverse external relationships that are at the core of all knowledgebrokering efforts. 


\section{Addendum}

The Spastic Centre's knowledge-brokering efforts continue, with two recent initiatives using new digital communication and collaboration tools.

\section{ParentWise podcast series}

This series of podcasts for families blends research evidence and practical wisdom in a readily accessible form. Written and presented by people who have had many years working with children with a disability, these audiocasts cover such diverse topics as building networks of support, managing a child's behaviour, building resilience, assistive technology options, respite, mealtime issues, a child's growing awareness, siblings and nurturing relationships. The development of the podcasts required a careful brokering process to translate and merge research evidence, clinical practice and the authentic, lived experience of families who had a child with cerebral palsy. The audiocasts and transcripts are available on the web site of the Cerebral Palsy Foundation and on iTunes. CDs of the first series have been distributed to families across the organisation and preloaded iPods made available for loan. The project was made possible through funding by the NSW Department of Human Services, Ageing Disability and Home Care.

\section{Knowledge Hub wiki}

The development of a new organisational intranet presented an opportunity to implement a number of social media and collaboration tools. One of the intranet project's aims was to introduce staff to the concept of distributed publishing, in which content experts from around the organisation can create and maintain web site (intranet) pages.

To facilitate this, a wiki (MediaWiki) was integrated into the new intranet. This section, called 'The Knowledge Hub', was designed to gather together information resources but also to be a place where the outcomes of knowledgebrokering efforts could be shared across the organisation.

The first major section of the Knowledge Hub was an Evidence-Based Clinical Decision-Making Library. The project was instigated by The Spastic Centre's Allied Health Consultants and subsequently led and implemented by the centre's Cerebral Palsy Institute. It was designed to support staff at The Spastic Centre to make evidence-based clinical decisions and was part of a broader, multifaceted project to implement a new model of service delivery across the organisation: the Life Needs approach. The development of the content for this section involved a brokering process of collaboratively appraising and 
translating research evidence - to distil current knowledge in the areas of assessment, intervention and prognosis/prevalence and also develop a set of clinical algorithms. The resources are designed to guide staff to achieve the best possible outcomes, in partnership with clients and families. Studies examining the effectiveness of these approaches, using a randomised controlled trial design are now under way (Campbell et al. 2010).

\section{References}

Access Economics 2008, The economic impact of cerebral palsy in Australia 2007, April 2008, Report for Cerebral Palsy Australia, Box Hill, Vic., viewed 6 August 2008, <http://www.cpaustralia.com.au/news/access_economics_ report.pdf>

Australian Bureau of Statistics (ABS) 2009, Household Use of Information Technology, Australia, 2008-09, Australian Bureau of Statistics, Canberra, viewed 1 December 2009, <http://www.abs.gov.au/Ausstats/abs@.nsf/ $\mathrm{mf} / 8146.0>$

Australian Institute of Health and Welfare 2006, Therapy and Equipment Needs of People with Cerebral Palsy and Like Disabilities in Australia, Australian Institute of Health and Welfare, Canberra, viewed 1 June 2007, <http:// www.aihw.gov.au/publications/dis/tenpwcplda/tenpwcplda.pdf>

Baldry, E., Bratel, J., Durrant, M. and Dunsire, M. 2005, 'Keeping children with a disability safely in their families', Practice, vol. 17, no. 3, pp. 143-56.

Brafman, O. and Beckstrom, R. 2006, The Starfish and the Spider: The unstoppable power of leaderless organizations, Penguin Books, New York.

Campbell, L., Novak, I. and McIntyre, S. 2010, 'Patterns and rates of use of an evidence-based practice intranet resource for allied health professionals: a randomised controlled trial', Developmental Medicine and Child Neurology, vol. 52, no. S2, p. 31.

Canadian Health Services Research Foundation 2003, The Theory and Practice of Knowledge Brokering in Canada's Health System, Canadian Health Services Research Foundation, Ottowa, viewed 10 July 2007, http://www.fcrss.org/ brokering/pdf/Theory_and_Practice_e.pdf

Canadian Health Services Research Foundation n.d., Knowledge Brokering, Canadian Health Services Research Foundation, Ottawa, viewed 10 July 2007, <http://www.chsrf.ca/keys/use_knowledge_e.php> 
Centre for Corporate Public Affairs 2007, Corporate Community Investment in Australia, Centre for Corporate Public Affairs, Canberra.

Clark, G. and Kelly, E. 2005, New Directions for Knowledge Transfer and Knowledge Brokerage in Scotland, Office of the Chief Researcher, Scottish Executive, Edinburgh, viewed 10 July 2007, <http://www.scotland.gov.uk/Resource/ Doc/69582/0018002.pdf>

ComScore 2008, 'Top social networking sites by unique visitors, May 2008', ClickZ, viewed 10 July 2008, <http://www.clickz.com/showPage. html?page $=3629976>$

Cusick, A., Convey, M., Novak, I. and McIntyre, S. 2009, ‘Employer-sponsored occupational therapy professional development in a multi-campus facility: a quality project', Australian Occupational Therapy Journal, vol. 56, pp. 22938.

Denning, S. 2006, 'Effective storytelling: strategic business narrative techniques', Strategy \& Leadership, vol. 34, no. 1, pp. 42-8.

Forkner-Dunn, J. 2003, 'Internet-based patient self-care: the next generation of health care delivery', Journal of Medical Internet Research, vol. 5, no. 2 (April-June), viewed 7 June 2007, <http://www.pubmedcentral.nih.gov/ articlerender.fcgi?artid $=1550561>$

Friedman, T. 2005, The World is Flat: A brief history of the twenty-first century, Farrar, Straus and Giroux, New York.

Greenhalgh, T., Robert, G., Macfarlane F., Bate, P. and Kyriakidou, O. 2004, 'Diffusion of innovations in service organizations: systematic review and recommendations', Milbank Quarterly, vol. 82, no. 4, pp. 581-629, viewed 7 June 2007, <http://www.milbank.org/quarterly/8204feat.html>

Kidwell, J., Vander Linde, K. and Johnson, S. [PricewaterhouseCoopers LLP] 2000, Applying corporate knowledge management practices in higher education', EDUCAUSE Quarterly Articles, viewed 7 June 2007, <http:// connect.educause.edu/Library/EDUCAUSE+Quarterly/ApplyingCorporateK nowledg/39668?time $=1218082810>$

Novak, I., McIntyre, S., Sharp, N. and Porter, J. 2008, 'Education with employer support improves allied health evidence-based practice knowledge and implementation in a cerebral palsy specialist service', Developmental Medicine and Child Neurology, vol. 50, s. 113, p. 43. 
Novak, I. and McIntyre, S. (forthcoming), 'Education with workplace supports improves practitioners' evidence based practice knowledge and implementation behaviours', Australian Occupational Therapy Journal.

Sifry, D. 2007, 'The state of the live Web, April 2007', Sifry's Alerts, viewed 7 June 2008, <http://www.sifry.com/alerts/archives/000493.html>

Sillence, E., Briggs, P. and Fishwick, L. 2005, 'Guidelines for developing trust in health websites', Proceedings of the International World Wide Web Conference [poster session], Chiba, Japan, pp. 1026-7, viewed 7 June 2008, ACM Digital Library.

Sillence, E., Briggs, P., Harris, P. and Fishwick, L. 2006, 'Changes in online health usage over the last 5 years', Proceedings of the 2006 Conference on Human Factors in Computing Systems, CHI 2006, Montréal, Québec, 22-27 April 2006, ACM 2006, pp. 1331-6, viewed 7 June 2008, ACM Digital Library.

Snyder, J., Carpenter, D. and Slauson, G. 2006, MySpace.com-a social networking site and social contract theory, Paper presented at 2006Proc ISECON conference, Dallas, Texas, viewed 1 March 2008, < http://isedj.org/ isecon/2006/3333/ISECON.2006.Snyder.pdf> 